\title{
Observation of the geometric amplitude factor in an optical system
}

\author{
D. BOUWMEESTER $\uparrow$, G. P. KARMAN, N. H. DEKKER †, \\ C. A. SCHRAMA§ and J. P. WOERDMAN \\ Huygens Laboratory, University of Leiden, \\ P.O. Box 9504, 2300 RA Leiden, The Netherlands
}

(Received 15 January 1996)

\begin{abstract}
By manipulating the discrete optical levels inside an optical resonator, we obtain a classical realization of a twisted Landau-Zener model. We experimentally demonstrate the geometric amplitude factor in the transition amplitude that arises for this model. We consider in particular the region of parameter space addressed in the original study of the geometric amplitude factor by M. V. Berry [7].
\end{abstract}

\section{Introduction}

In previous papers we have demonstrated that the quantum-mechanical description of a coupled two-level system also applies to macroscopic optical two-level systems [1-6]. The discrete levels correspond to resonance conditions, the optical field inside the resonator can be mapped onto the quantum-mechanical wave function, and the evolution of the optical field is approximately governed by a Schrödinger equation. The macroscopic nature of the optical two-level system provides some advantages over quantum-mechanical two-level systems. First, a precise control of the various parameters is possible. Second, a continuous measurement can be performed on the classical optical field without influencing its dynamics, whereas in the quantum case a measurement yields a collapse of the wavefunction. These advantages enable an experimental study of topics in quantum physics that have received almost exclusively theoretical attention.

One of these topics deals with the geometric amplitude factor in quantum transitions, as predicted by Berry in 1990 [7] and further elaborated upon by Nakamura and Rice [8,9]. Similar theoretical predictions were independently made by Joye, Mileti, Pfister and Kunz [10,11]. Up to now only one experiment has been reported, performed on a nuclear spin- $\frac{1}{2}$ system, which demonstrates some features of the geometric amplitude factor [12].

The geometric amplitude factor is a direct consequence of the geometric phases that arise due to the curvature of the path followed in parameter space [13, 14]. Whereas the geometric phase is usually considered for a closed path in parameter space, the geometric amplitude factor arises for avoided-crossing models for which

$\uparrow$ Present address: Oxford University, Mathematical Institute, 24-29 St. Giles, Oxford OX1 3LB, United Kingdom.

+ Present address: Harvard University, Jefferson Laboratory, 17a Oxford St. Cambridge, MA 02138 , USA.

$\S$ Present address: NMI Laboratory, P.O. Box 654, 2600 AR Delft, The Netherlands. 
the corresponding path is open. The reason why the geometric phase is only unambiguously defined for a closed path is that only a phase difference is physically relevant. The geometric phase is then defined as the difference between the initial phase of the system and the final phase after the control parameters of the system have returned to their initial values and after substracting the dynamical phase. At first sight it seems, therefore, impossible that a phase of geometric origin gives rise to an observable effect for an open path. However, one should realize that if the open path represents an avoided-crossing model it leads to transition dynamics that depend on the relative differences between the two adiabatic eigenstates. Since curvature in the path followed in parameter space induces opposite geometric contributions to the phases of the two adiabatic states, a relative geometric phase difference is locally defined. If this geometric phase difference changes as function of time then observable effects can arise in the transition probability.

Throughout the theoretical presentation of the geometric amplitude factor we will use the familiar quantum-mechanical formulation in which $h$ appears. Our experiments are, however, performed on classical optical systems for which optical frequency levels play the role of quantum-mechanical energy levels. Using the relation $E=h v$ (where $E$ is energy, $v$ is frequency and $h$ the Planck constant) allows for a simple transformation of the quantum-mechanical formulation to the classical formulation. Throughout the experimental presentation we will use the familiar optical notion in terms of frequencies.

In order to give a clear presentation of the geometric amplitude factor, as introduced by Berry [7], we briefly review in section 2 the mathematical formulation of the geometric phase and describe in section 3 the elegant method developed by Dykhne [15], and by Davis and Pechukas [16], which we refer to as the DDP method, for calculating transition probabilities. In section 4 the useful relation between geometric effects and dynamical effects is reviewed. This relation, in combination with the DDP method, provides clear insight into the consequences of the geometric amplitude factor. In section 5 we introduce an experimentally realizable twisted Landau-Zener model for which a geometric amplitude factor is expected to arise. The optical implementation of this specific model is described in section 6. The experimental results, in the parameter region where our model closely resembles the model proposed by Berry [7], are presented in section 7. Conclusions are drawn in section 8 .

\section{Geometric phase}

Throughout this article we will exclusively consider the case of a two-level system characterized by the Hamiltonian

$$
\hat{\mathbf{H}}(t)=\left[\begin{array}{cc}
Z(t) & X(t)-\mathrm{i} Y(t) \\
X(t)+\mathrm{i} Y(t) & -Z(t)
\end{array}\right] .
$$

The parameter space has three coordinates $X, Y, Z$, and the path $\gamma$, followed in parameter space is given by $(X(t), Y(t), Z(t))$. An expression for the geometric phase is obtained starting from the Schrödinger equation:

$$
\mathrm{i} h \frac{\mathrm{d}}{\mathrm{d} t}|\Psi(t)\rangle=\hat{\mathbf{H}}(R(t))|\Psi(t)\rangle .
$$

Here, $R(t)$ indicates that the Hamiltonian depends on a set of time-dependent 
parameters. We assume that at $t=0$ the two-level system is in one of the two adiabatic eigenstates, labelled with + or - ,

$$
|\Psi(0)\rangle=| \pm, R(0)\rangle \text {. }
$$

Under the assumption of adiabatic following, the wavefunction evolves as:

$$
|\Psi(t)\rangle=\exp \left\{-\frac{\mathrm{i}}{\hbar} \int_{0}^{t} E_{ \pm}(R(s)) \mathrm{d} s+\mathrm{i} \gamma_{ \pm}(t)\left|\frac{t}{h}, R(t)\right\rangle,\right.
$$

where $E_{ \pm}$are defined by

$$
E_{ \pm}= \pm\left[X^{2}(t)+Y^{2}(t)+Z^{2}(t)\right]^{1 / 2}
$$

The first term in the exponent of equation (4) is i times the dynamical phase and the second term is i times the geometric phase [13]. The latter can be expressed as

$$
\begin{aligned}
\gamma_{ \pm}(t) & =\mathrm{i} \int_{0}^{t}\left\langle \pm, R(s)\left|\frac{\mathrm{d}}{\mathrm{d} s}\right| \pm, R(s)\right\rangle \mathrm{d} s \\
& = \pm \frac{1}{2} \int_{0}^{t} \frac{(X Y-Y X) Z}{\left(X^{2}+Y^{2}\right)\left(X^{2}+Y^{2}+Z^{2}\right)^{1 / 2}} \mathrm{~d} s,
\end{aligned}
$$

where the overdots denote derivatives with respect to time. For later use, it is convenient to express $\hat{\mathbf{H}}(t)$ and $\gamma_{ \pm}$in polar coordinates $E, \theta, \phi$,

$$
\begin{aligned}
\hat{\mathbf{H}}(t) & =E(t)\left[\begin{array}{cc}
\cos \theta(t) & \sin \theta(t) \exp (-\mathrm{i} \phi(t)) \\
\sin \theta(t) \exp (+\mathrm{i} \phi(t)) & -\cos \theta(t)
\end{array}\right], \\
\gamma_{ \pm}(t) & = \pm \frac{1}{2} \int_{0}^{t} \cos \theta(s) \dot{\phi}(s) \mathrm{d} s
\end{aligned}
$$

The function $\phi(t)$ will play an important role in this paper and we will refer to it as the twist function.

As mentioned above, the geometric phase is usually written in the form of a closed circuit integral in parameter space. Such an expression explicitly indicates that the geometric phase is a global quantity and that it is independent of how fast the path in parameter space is followed. In this paper, however, local aspects of the geometric phase are essential and therefore an expression in the form of equation (6) is convenient. Unlike the closed circuit expression for the geometric phase, equation (6) is not explicitly time independent. A simple way to see if an expression obtained by an open-path integral is independent of how fast the path is followed is to replace $t$ by a scaled time $\tau \equiv \delta t$. We will refer to $\delta$ as the scaled-time parameter. If an expression is independent of $\delta$ it is clearly independent of how fast the path is followed; this is the often-used criterion for a geometric effect. We will use the scales time $\tau$ throughout the rest of this paper.

\section{The Dykhne-Davis-Pechukas method}

The adiabatic assumption, crucial for the derivation of the geometric phase, seems to impose a severe restriction on the application of the above theory. However, the DDP method allows transitions, i.e. diabatic dynamics, to be described under the adiabatic assumption [15-17]. Therefore, the DDP method 
provides a way of including the effect of geometric phases on the transition properties [7, 9-11].

The DDP method is based on the adiabatic assumption that coupling between the adiabatic energy levels can only occur at positions where the adiabatic energies are degenerate. In the case of avoided-crossing models the adiabatic energy levels are degenerate at complex time points $\tau_{0}$. For example in the case of the conventional Landau-Zener model $[18,19]$, characterized by $X(\tau)=\Delta, Y(\tau)=0$, and $Z(\tau)=\alpha \tau$, the adiabatic energies and the complex time points where degeneracies occur are given by

$$
\begin{aligned}
& E_{ \pm}= \pm\left(\alpha^{2} \tau^{2}+\Delta^{2}\right)^{1 / 2}, \\
& \tau_{0}= \pm \mathrm{i} \Delta /|\alpha| .
\end{aligned}
$$

An adiabatic energy surface corresponding to a two-level system is usually defined through the square root of a complex function, as is the case in equation (10), and is therefore a double-valued function. To emphasize the mathematical properties of such functions, consider the generic case of

$$
f(z)=z^{1 / 2}=r^{1 / 2} \exp (\mathrm{i} \psi / 2) .
$$

Clearly $z$ remains unchanged if $\psi$ is replaced by $\psi+2 \pi$ whereas $z^{1 / 2}$ changes sign. In this situation, it is common to introduce a Riemann surface which is a generalization of the complex plane to a surface of two Riemann sheets. On the Riemann surface $f(z)$ is single-valued. The two Riemann sheets are connected by a cut starting at the zero point $\tau_{0}$ of the square root. As a point $z$ starts on one Riemann sheet and describes a continuous circuit around $\tau_{0}$ it passes to the other Riemann sheet when it crosses the cut. The point $\tau_{0}$ is referred to as a branch point. The real part of the adiabatic energy surfaces $E_{ \pm}$, given in equation (10), are the two sheets of the Riemann surface draw in figure 1.

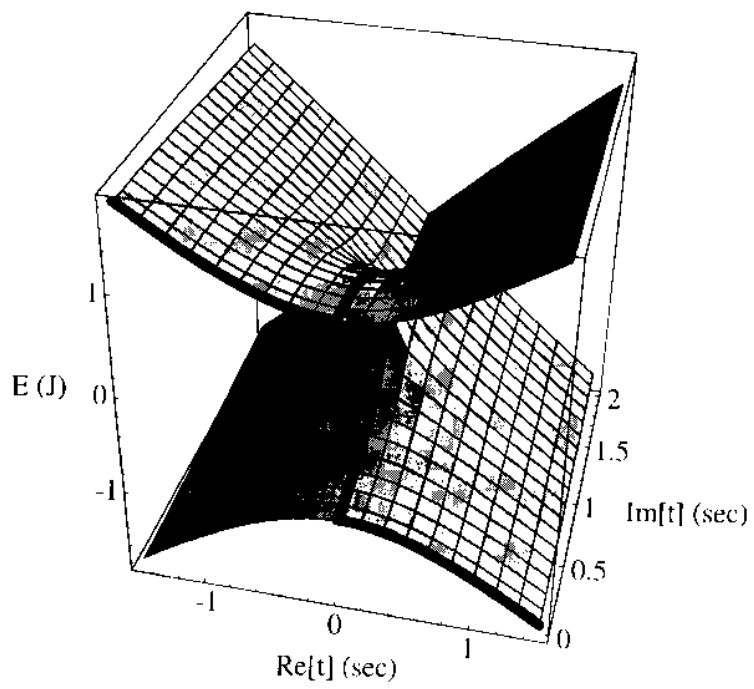

Figure 1. Real part of the adiabatic energy surface for (positive imaginary) complex times. The parameters used are $\alpha=1 \mathrm{~J} \mathrm{~s}^{-1}, \Delta=1 \mathrm{~J}$, and $\delta=1$. The front surface of the plot shows the familiar avoided crossing curves for real times. The thick curve indicates the path of integration used in the Dykhne-Davis-Pechukas method. 


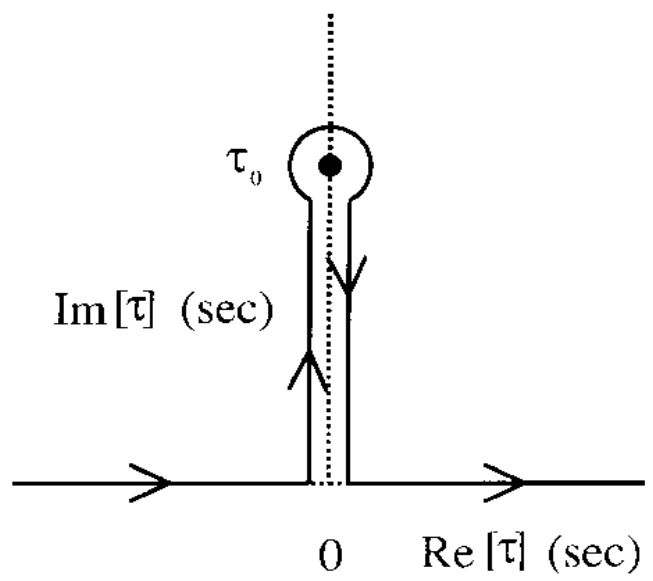

Figure 2. Illustration of the Dykhne-Davis-Pechukas method. This graph is a schematic representation of the thick curve in figure 1 .

As mentioned before, for transitions to occur under the adiabatic assumption, $|\Psi(\tau)\rangle$ must ' reach' a branch point around which the transition between the adiabatic energy levels takes place. Subsequently $|\Psi(\tau)\rangle$ must return to the real time axis as indicated in figure 1 by the thick curve. A schematic drawing of this curve is shown in figure 2.

If we assume as initial state one of the adiabatic eigenstates, say $|+\rangle$, then equation (4) for the adiabatic 'evolution' of $|\Psi(\tau)\rangle$ from $\tau=0$ to the branch point $\tau=\tau_{0}$ reads

$$
\left|\Psi\left(\tau_{0}\right)\right\rangle=\exp \left\{-\frac{\mathrm{i}}{\hbar \delta} \int_{0}^{\tau_{0}} \mathrm{~d} \tau\left[E_{+}(\tau)+\frac{\hbar \delta}{2} \dot{\phi} \cos \theta\right]|\xi\rangle .\right.
$$

Since the time is complex valued, the exponent in equation (13) no longer represents a pure phase factor; instead is also represents damping if the branch point is chosen in the lower half of the complex-time plane.* Subsequent integration along an infinitesimally small circle around the branch point accomplishes the change from the one adiabatic energy (Riemann) sheet to the other. Integration along the return path to the real time axis yields an expression identical to equation (13) since exchange of the integration boundaries and change of the adiabatic energy sheets both give a multiplication factor -1 . An additional factor of 2 in the exponent is needed to obtain from the total transition amplitude the expression for the total transition probability $P$,

$$
P \approx \exp \left\{-\Gamma_{\mathrm{d}}\right\} \exp \left\{+\Gamma_{\mathrm{g}}\right\}
$$

Here the dynamical amplitude factor is given by

$$
\Gamma_{\mathrm{d}}=-\frac{4}{\hbar \delta} \operatorname{Im} \int_{0}^{\tau_{0}} \mathrm{~d} \tau E(\tau)
$$

* The branch points in the lower-half of the complex-time plane should be used if transitions from the higher energy levels to the lower energy levels are considered. The branch points in the upper-half of the complex-time plane should be used in the opposite case. 
and the geometric amplitude factor by

$$
\Gamma_{\mathrm{g}}=-2 \operatorname{Im} \int_{0}^{\tau_{0}} \mathrm{~d} \tau \dot{\phi} \cos \theta .
$$

We note that the dynamical (geometric) amplitude factor arises from the analytical continuation of the dynamical (geometric) phase into the complex-time plane. We stress that equation (14) is an approximate equality since the DDP method has been only rigorously proven in the adiabatic limit, i.e. for small $\delta$. It is, however, well known that the DDP method happens to provide the exact expression in the case of the conventional Landau-Zener model.

\section{Geometric versus dynamical amplitude factors}

Note that the adiabatic energies of the general two-level Hamiltonian [equation (8)] are independent of the twist function $\phi(\tau)$. Hence the branch points will also be independent of the curvature of the path in parameter space induced by $\phi(\tau)$.

It is possible to relate the geometric amplitude factor to the familiar dynamical amplitude factor by performing the following unitary transformation [7].

$$
\begin{aligned}
|\Psi(\tau)\rangle & =\hat{\mathbf{U}}(\tau)|\Psi(\tau)\rangle \\
& \equiv\left[\begin{array}{cc}
\exp (-\mathrm{i} \phi(\tau) / 2) & 0 \\
0 & \exp (+\mathrm{i} \phi(\tau) / 2)
\end{array}\right]|\Psi(t)\rangle .
\end{aligned}
$$

The corresponding Hamiltonian is given by

$$
\begin{aligned}
\hat{\mathbf{H}}^{\prime}(\tau) & =\hat{\mathbf{U}}^{\dagger} \hat{\mathbf{H}} \hat{\mathbf{U}}-\mathrm{i} \hbar \delta \hat{\mathbf{U}}^{\dagger} \hat{\mathbf{U}} \\
& =\left[\begin{array}{cc}
Z-\frac{1}{2} \hbar \delta \dot{\phi} & \left(X^{2}+Y^{2}\right)^{1 / 2} \\
\left(X^{2}+Y^{2}\right)^{1 / 2} & -\left(Z-\frac{1}{2} \hbar \delta \dot{h}\right)
\end{array}\right] \\
& =\left[\begin{array}{rr}
Z^{\prime} & X^{\prime} \\
X^{\prime} & -Z^{\prime}
\end{array}\right] .
\end{aligned}
$$

$\hat{\mathbf{H}}^{\prime}$ is now real symmetric instead of complex hermitian. This yields a zero geometric phase and geometric amplitude in the primed frame. The twist function, $\phi(\tau)$, now manifests itself in the adiabatic energy levels

$$
E_{ \pm}^{\prime}= \pm\left[\left(Z-\frac{1}{2} h \delta \dot{\phi}\right)^{2}+X^{2}+Y^{2}\right]^{1 / 2}
$$

Since the transformation matrix $\hat{\mathbf{U}}$ between $|\Psi(t)\rangle$ and $|\Psi(t)\rangle$ contains only phase factors on its diagonal elements, the transition probability between the adiabatic levels in the primed frame is identical to that in the unprimed frame.

The advantage of the transformation to the primed frame is that the geometric effects, induced by $\phi(\tau)$, are expressed as shifts and splittings of the branch points. Shifts of branch points towards or away from the real time axis indicate an increase or decrease, respectively, of the transition amplitude. Splitting of the branch points indicates that the particular model behaves as a sequence of conventional Landau-Zener crossings. The extension of the DDP method to the case that several pairs of branch points influence the transition dynamics can be found in references $[11,20,21]$. 
At this point we would like to make a critical comment regarding the experiments of Zwanziger, Rucker, and Chingas [12], which were claimed to be a demonstration of the geometric amplitude factor. In that work, transformation (17), which is a mathematical tool to compare the geometric effects to the familiar dynamical effects, has been taken to the experimental level; a conventional Landau-Zener experiment has been performed where $\alpha \tau$ is simply replaced by $(\alpha \tau-1 / 2 \hbar \delta \dot{\phi})$ and which is analyzed as if it originated from a system characterized by a complex hermitian (twisted Landau-Zener) Hamiltonian. Therefore the experiment was in essence a conventional Landau-Zener experiment. In fact, it is straightforward to formulate the optical variety of this approach $\uparrow$.

\section{Twisted Landau-Zener models}

We consider avoided-crossing models which are characterized by the following Hamiltonian:

$$
\hat{\mathbf{H}}_{\text {twist }}=\left[\begin{array}{cc}
\alpha \tau & \Delta \exp \{-\mathrm{i} \phi(\tau)\} \\
\Delta \exp \{+\mathrm{i} \phi(\tau)\} & -\alpha \tau
\end{array}\right] .
$$

We refer to such models as twisted or winding Landau-Zener models [7, 9]. Berry analyzed, in particular, the case that [7]

$$
\phi(\tau)=\beta \tau^{2}
$$

According to equations (15) and (16) the dynamical amplitude factor and the geometric amplitude factor are given by

$$
\begin{aligned}
& \Gamma_{\mathrm{d}}=\pi^{2} /(\hbar \delta|\alpha|), \\
& \Gamma_{\mathrm{g}}=-\left(\pi \beta \Delta^{2} / \alpha^{2}\right) \operatorname{sgn}(\alpha) .
\end{aligned}
$$

Recall that both expressions are approximations valid for small $\delta$. Note that $\Gamma_{\mathrm{g}}$ is independent of $\delta$ and $h$ which is usually taken as the characteristic feature of a geometric effect. In the primed frame, the branch points of equation (19) read for this particular model

$$
\tau_{0}^{\prime}= \pm \mathrm{i} \Delta /|\alpha-\hbar \hbar \delta \beta|
$$

The branch points have been shifted with respect to the branch points of the conventional Landau-Zener (LZ) model [equation (11)]; this indicates a change in the transition amplitude. The exact expression for the transition probability in the primed frame contains only a dynamical amplitude factor

$$
\Gamma_{\mathrm{d}}=\not a^{2} /(h \delta \mid(\alpha-\hbar) \delta \beta) \mid \text {. }
$$

This model illustrates in the simplest way the geometric amplitude factor however; it has the unphysical property of diverging values for $\phi(\tau)$ as $\tau \rightarrow \pm \infty$.

$\dagger$ The optical variety of the experimental approach of reference [12] is as follows. Consider an optical resonator with only two EOM's and perform an conventional LandauZener experiment with $\alpha t$ replaced by $(\alpha t-1 / 2 \hbar \dot{\phi})$ [5]. The light leaking out of the cavity can be analysed after performing the unitary transformation given in equation (17) by an additional EOM outside the resonator. Since this EOM must be placed with its axis along the detection axis of the polarization it cannot alter the population of the detected polarization. Therefore this EOM can be omitted in the experiment. What remains is the conventional dynamical Landau-Zener experiment. 


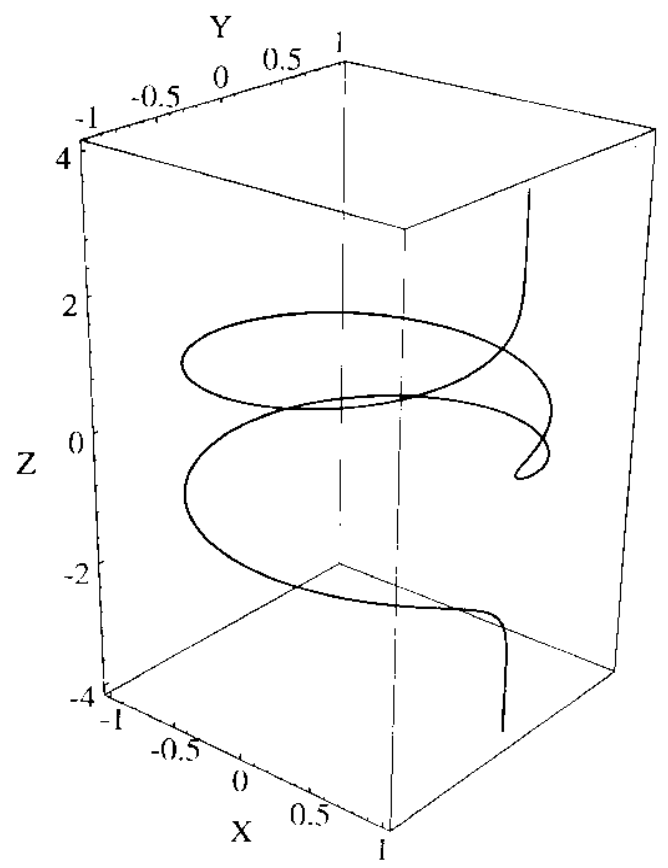

Figure 3. Plot of the Hamiltonian curve for the Gaussian twisted Landau-Zener model $(\mu=4.5 \mathrm{rad}, a=1 \mathrm{~s}, \delta=1)$.

From an experimental point of view, we are interested in twisted LZ models with finite values for $\phi(\tau)$ as $\tau \rightarrow \pm \infty$. We have performed our experiments by implementing the following model:

$$
\phi(\tau)=\mu\left(1-\exp \left\{-(\tau / a)^{2}\right\}\right),
$$

which approaches the $\phi(\tau)=\beta \tau^{2}$ model near the avoided crossing at $\tau=0$, but approaches the constant value $\mu$ for $\tau \rightarrow \pm \infty$. The path in parameter space corresponding to this model $(\mu=4.5 \mathrm{rad}, a=1 \mathrm{~s}, \delta=1)$ is drawn in figure 3 . We will refer to this model as the Gaussian twisted LZ model.

This model has many pairs of branch points for equation (19) in the primed frame; however, only those for which the amplitude factor $\Gamma_{\mathrm{d}}$ is relatively small will contribute significantly to the transition amplitude. For our model these dominant points are the ones closest to the real-time axis $\$$. Their positions are obtained most easily numerically. However, to gain some insight it is useful to present first some analytical considerations regarding their positions.

The conventional LZ model has branch points on the imaginary time axis [see equation (11)] which indicates that the position of nearest approach of the two levels is at $\tau=0$. If we assume that the addition of a twist function gives rise to small changes only, it is expected that the dominant branch points remain on the imaginary time axis. Therefore we insert $\tau=\mathrm{i} \xi$, with $\xi$ real and $\delta=1$, into

\$ There exist crossing-models for which the dominant branch points are not those closest to the real-time axis $[10,11]$. 

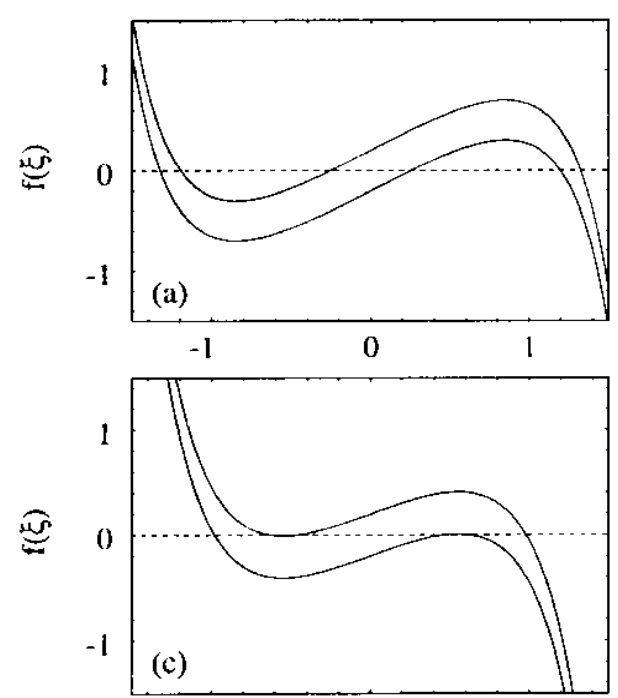

$-1$

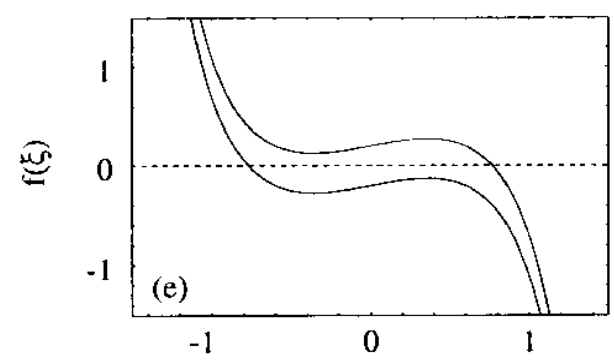

$\xi$ (sec.)
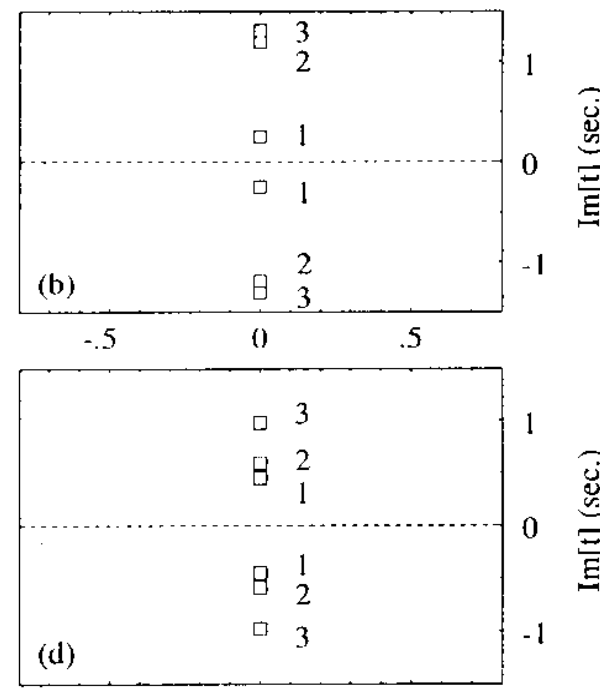

Ej
E
E

Figure 4. Graphs (a), (c), and (e) are plots of $f(\xi)$ for $\mu \hbar=0 \cdot 2,0 \cdot 46,0 \cdot 7 \mathrm{~J} \mathrm{~s}$. The solutions for $f(\xi)=0$ indicate branch points on the imaginary time axis. Graphs $(b),(d)$, and $(f)$ show the branch points corresponding to graphs $(a)$, $(c)$, and (e), respectively. Note that pairs 1 and 2 in graph $(f)$ cannot be determined by graph $(e)$ but have been obtained by numerical calculations.

equation (19), with $\phi(\tau)$ given by equation (26). The branch points (determined by $E_{ \pm}^{\prime}=0$ ) on the imaginary axis are i times the solutions for $\xi$ of

$$
f(\xi) \equiv \alpha \xi-\hbar \mu\left(\xi / a^{2}\right) \exp \left\{(\xi / a)^{2}\right\} \pm \Delta=0 .
$$

The function $f(\xi)$ is plotted in figures $4(a)$, (c) and (e), for increasing magnitude $\mu$ of the twist function ( $\alpha=1 \mathrm{~J} \mathrm{~s}^{-1}, a=1 \mathrm{~s}, \delta=1$, and $\left.\Delta=0 \cdot 2 \mathrm{~J}\right)$. The corresponding branch points are plotted in figures $4(b),(d)$ and $(f)$.

For $h \mu=0.20 \mathrm{~J} \mathrm{~s}$, shown in figures $4(a)$ and $(b)$, pair 1 of the branch points will dominate the transition amplitude. The additional pairs 2 and 3 are the consequence of the physical requirement that $\phi(\tau)$ is finite for $\tau= \pm \infty$. Since both pairs 2 and 3 have large imaginary values they have negligible influence on the transition amplitude. In this situation the Gaussian twisted LZ model is a good 
approximation to the $\phi(\tau)=\beta \tau^{2}$ model, and is therefore suited to study the geometric amplitude factor as described by Berry [7].

If the magnitude of the twist function is increased another interesting situation arises. First, the pairs 1 and 2 of the branch points approach each other as shown in figures $4(c)$ and $(d)$, for $h \mu=0.46 \mathrm{~J} \mathrm{~s}$. Next, as shown in figure $4(e)$ and $(f)$, for $h \mu=0.70 \mathrm{~J} \mathrm{~s}$, pairs 1 and 2 disappear from the imaginary axis as can be seen from the reduction from 6 to 2 solutions for $f(\xi)=0$ in figure 4 (e). By numerical calculations one finds that these pairs move away from each other keeping equal distance to the real time axis. The transition amplitude will now be dominated by three pairs of branch points and interference phenomena similar to those arising for a sequence of three conventional LZ crossings are expected. In this situation the influence of the twist function can no longer be described by a geometric amplitude factor but manifests itself in an intertwined way with the dynamical amplitude factor [23].

\section{Optical realization}

For our experiments we used an optical two-level system which is schematically shown in figure 5. The two levels are formed by two orthogonal polarization states of a single longitudinal mode of a 7-meter long ring cavity. The degeneracy in frequency of the two polarizations is lifted by an electro-optic modulator (EOM1) which consists of a birefringent crystal. The birefringence is controlled by applying an electric voltage over the crystal. Including a second electro-optic modulator (EOM2) under $45^{\circ}$ with respect to EOM1 provides coupling between the two polarizations.

The optical two-level system is prepared in its initial state by injecting linearly polarized light from a single-frequency HeNe laser. As soon as the injection light is

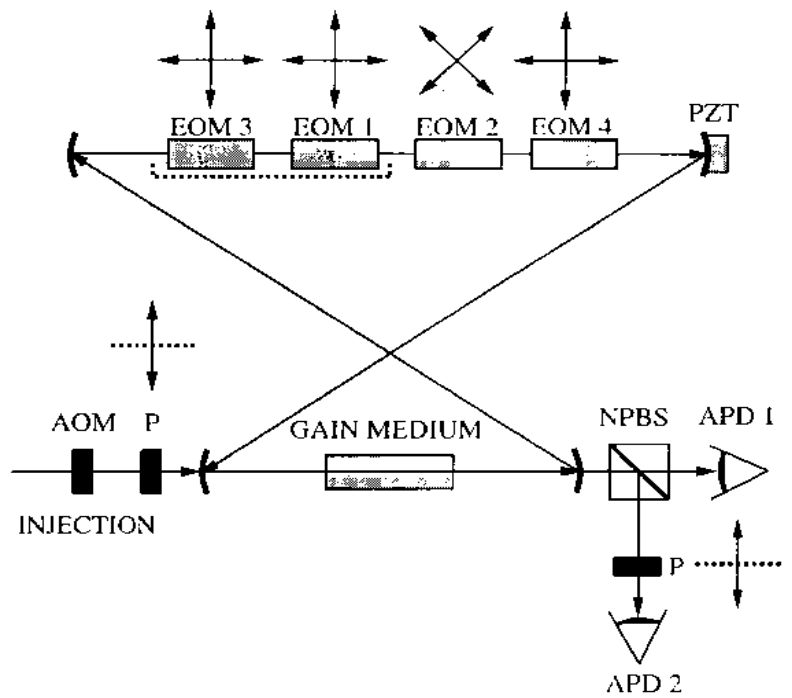

Figure 5. Schematic drawing of the optical part of the setup. The acronyms used are AOM for acousto-optic modulator, $P$ for polarizer, EOM for electro-optic modulator, PZT for piezo element, NPBS for non-polarizing beam splitter, and APD for avalanche photo diode. The dotted line below EOM1 and EOM 3 indicates that they have been combined into one EOM in the actual experiments. 
resonant with the ring cavity (which is slowly scanned in length by a piezo element (PZT) mounted behind one of the cavity mirrors), the light intensity builds up inside the ring cavity. At a certain intracavity intensity the injection light is switched off within $10 \mathrm{~ns}$ using an acousto-optic modulator (AOM). Due to optical losses of the EOMs and the mirrors, the light intensity will decrease after switching off the injection, with a characterized cavity decay time $t_{\text {cav }}$. Within $t_{\text {cav }}$ we must perform our experiments, i.e., drive the EOMs with the proper time-dependent voltages (up to $400 \mathrm{~V}$ ) and analyse the small fraction of the light intensity that leaks through one of the cavity mirrors. In order to make such experiments electronically feasible $t_{\text {cav }}$ must be at least a few $\mu$; for our cavity length this corresponds to a cavity finesse $\mathscr{F}$ of the order of $10^{3}$. To obtain such a high cavity finesse the optical losses inside the cavity must be compensated for. Therefore we designed a polarization-independent $\mathrm{He}-\mathrm{Ne} 633 \mathrm{~nm}$ amplifier tube. This tube has anti-reflection coated windows (AR@633nm and AR@3.39 $\mu$ m) instead of Brewster windows. Operating the optical ring system closely below threshold provides a cavity decay time of approximately $10 \mu \mathrm{s}$; this is sufficiently large for the experiments $(\mathscr{F} \approx 1300)$. Of course, this optical amplification scheme would not be useful in the limit of a few photons in the ring since the amplifying medium will always contribute, on average, one spontaneously emitted photon to the optical cavity mode, thus randomizing the optical field both in phase and in polarization. However, we deal with relatively high optical intensities (of the order of $1 \mathrm{~mW}$ ); hence it is legitimate to neglect the influence of spontaneous emission.

In a previous paper [5] we demonstrated that the conventional LZ model can be realized in an optical two-level system by applying a linear voltage sweep to EOM1 and keeping the voltage across EOM2 constant. The mapping of the polarization state vector in the ring cavity to the quantum-mechanical wavefunction for a two-level system is obtained using Jones calculus [22]. For further details concerning this method and the experimental realization of an optical two-level system we refer to our previous papers $[5,6]$.

The Hamiltonian of current interest, given by equation (20), can be generated from the conventional LZ Hamiltonian by the following matrix multiplication:

$$
\mathbf{H}_{\mathrm{twist}}=\mathbf{M}(t)\left[\begin{array}{cc}
\alpha t & \Delta \\
\Delta & -\alpha t
\end{array}\right] \mathbf{M}(t)^{-1},
$$

where

$$
\mathbf{M}(t)=\left[\begin{array}{cc}
\exp (-\mathrm{i} \phi(t) / 2 & 0 \\
0 & \exp (+\mathrm{i} \phi(t) / 2)
\end{array}\right] .
$$

The matrices $\mathbf{M}(t)$ and $\mathbf{M}(t)^{-1}$ are physically obtained by means of additional birefringent elements, EOM3 and EOM4, their axes being parallel to those of EOM1 (see figure 5). The three control parameters are the voltages applied to the EOMs $\left[\alpha t \propto V_{\text {EOM } 1}, \Delta \propto V_{\text {EOM } 2}\right.$ and $\left.\phi(t) \propto V_{\text {EOM } 3}=-V_{\text {EOM } 4}\right]$. We remind the reader that it is essential to have three control parameters; a simpler setup, using only two control parameters, is inadequate. Note that we do indeed create $\mathbf{H}_{\text {twist }}$ as given by equation (20) instead of merely performing transformation (18). The polarization state of the light leaking out of the cavity is analysed by two avalanche photo diodes (APD) and is expected to provide a direct observation of the geometric amplitude factor. 
There are two complications of the current experiments compared to the previously reported LZ experiments [5]. First, it becomes necessary to employ a unidirectionally passed ring cavity instead of a bidirectional standing-wave cavity. The reason for this is that the precise ordering of the EOMs is of crucial importance to generate the twisted LZ model, whereas for the conventional LZ model the ordering is irrelevant. In other words, we explicitly use the fact that differently oriented EOMs do not commute. The disadvantage of using a ring cavity is that the alignment of the EOMs becomes very difficult, since the EOMs tend to slightly deviate the light path by an amount proportional to their electrical birefringence. In a standing-wave cavity such deviations are automatically corrected for since the light path reverses at the mirrors. To alleviate somewhat the alignment problem, we make use of the fact that EOM1 and EOM3 have their axes parallel and can be combined into one EOM.

The second complication is that more than one EOM must have a timedependent birefringence in order to generate the twisted LZ model. As a consequence, one should take into account retardation effects of the light propagating in between the optical elements [6]. The simplest way to overcome this complication is to place the time-dependent EOMs as close as possible to each other so that they operate approximately at the same position in the cavity.

\section{Experimental results}

The main experimental results presented in this article are in the parameter range for which the Gaussian twisted LZ model is a good approximation to the $\phi(\tau)=\beta \tau^{2}$ model treated by Berry [7]. This situation corresponds to figures $4(a)$ and $(b)$ where the contributions of the pairs of branch points 2 and 3 to the transition amplitudes is at least a factor $e^{-3} \approx 0.03$ smaller than the contribution of the dominant pair of branch points 1 . In other words, using the theoretical results for the $\phi(\tau)=\beta \tau^{2}$ model to analyse our Gaussian twisted LZ experiments should give an error of less than $3 \%$ in the transition amplitude. Figure 6 shows typical experimental data (the scaled-time parameter $\delta=1$ ). Curves 1,3 , and 5 are conventional $\mathrm{LZ}$ experiments in the diabatic, intermediate, and adiabatic limit, respectively. Curves 2 and 4 are experimental results for the Gaussian twisted LZ model (see equation (26)) with $a=0.6 \mu \mathrm{s}$, and $\mu=+1.52$ and $-1.52 \mathrm{rad}$, respectively; the values for $\alpha$ and $\Delta$ are the same as for curve $3\left(\alpha=1.65 \mathrm{MHz} \mu \mathrm{s}^{-1}\right.$, $\Delta=0.25 \mathrm{MHz}$ ). Each curve shows the time evolution of the normalized intensity of the polarization along the linear polarization of the injection light, say the + polarization.

Far before the avoided crossing, that is on the left-hand side of figure 6, all intracavity light is + polarized. The + and - polarizations correspond to the diabatic, i.e., the uncoupled, energy states of the system. Hence the experimental data provide a time trace of one of the diabatic populations. From this information the transition probability between the adiabatic eigenstates can be determined as follows. We use the fact that on the left- and right-hand side of figure 6 , far away from the avoided crossing at $t=0$, the adiabatic and the diabatic eigenstates coincide; they change labels at the avoided crossing. In the region near the avoided crossing the adiabatic eigenstates are superpositions of the + and - polarizations. If, after the avoided crossing, the two-level system is in a superposition of the adiabatic eigenstates, a beat frequency in both + and the - component of the polarization is present which increases in frequency and decreases in amplitude 


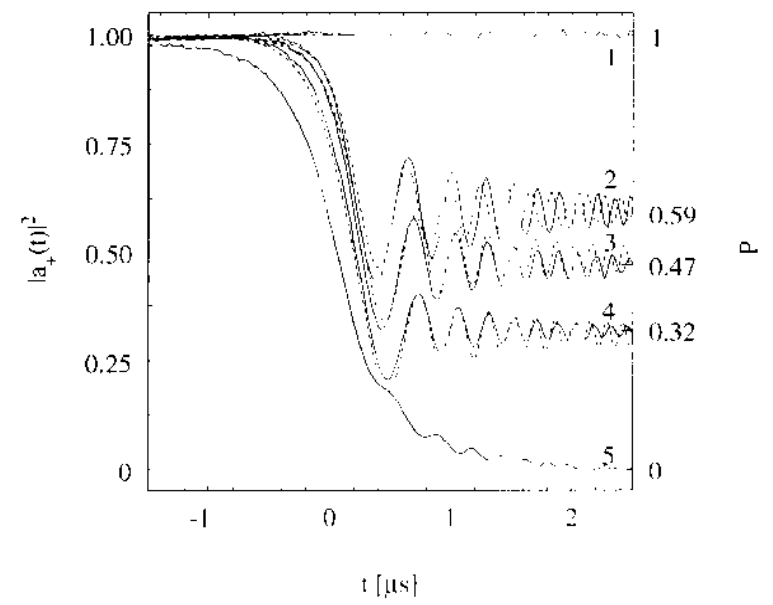

Figure 6. The solid curves are the experimentally obtained time traces for the normalized intensity of the + polarization. Each time trace corresponds to the average over 25 experimental runs. Curves 1,3 , and 5 are conventional Landau-Zener curves in the diabatic, the intermediate $\left(\alpha=1.65 \mathrm{MHz}_{\mu \mathrm{s}}^{-\mathrm{s}}, \Delta=0.25 \mathrm{MHz}\right)$, and the adiabatic limit, respectively. Curves 2 and 4 are experimental results for the Gaussian twisted Landau-Zener model for $\alpha=1.65 \mathrm{MHz}_{\mu}^{-1}, \Delta=0.25 \mathrm{MHz}, \mu= \pm 1.52 \mathrm{rad}$, and $a=0.6 \mu \mathrm{s}$. The dotted curves are the corresponding numerical simulations.

further away from the avoided crossing. To obtain the transition probability between the adiabatic energy levels we take the center of the oscillating time traces at the right-hand side of the graph, as indicated in figure 6.

The differences between the conventional LZ curve 3 and the two Gaussian twisted LZ curves 2 and 4 in figure 6 illustrate the significance of the geometric amplitude factor. We remind the reader that the adiabatic energy levels corresponding to curves 2,3 , and 4 are identical; the difference in the time traces is completely due to the twist function $\phi(\tau)$. Note that curves 2 and 4 have the opposite sign for $\phi(\tau)$.

To demonstrate that the geometric amplitude factor is independent of how fast the path in parameter space is followed, we performed a series of Gaussian twisted LZ experiments for different values for the scaled-time parameter $\delta$. In other words, we performed the same experiment faster and faster. In figure 7 we plot $-\ln P$, which is, according to equation (14), equal to $\Gamma_{\mathrm{d}}-\Gamma_{\mathrm{g}}$, as function of $1 / \delta$.

According to equations (22) and (23) we expect straight lines with slopes $\pi \Delta /|\alpha|$, and vertical offsets of $\Gamma_{\mathrm{g}}$ compared to the conventional LZ line. Line 2 is the experimental result for the conventional LZ model. Line 1 and 3 are the experimental results for the Gaussian twisted LZ model with opposite signs for the twist function. The three lines have an identical slope, $0 \cdot 98 \pm 0 \cdot 01$, in excellent agreement with the theoretical value of 0.98 . The offsets of curves 1 and 3 yield a geometric amplitude factor of $\Gamma_{\mathrm{g}}=-0 \cdot 23 \pm 0.01$ and $\Gamma_{\mathrm{g}}=0 \cdot 14 \pm 0 \cdot 01$, respectively. The theoretical values predicted in the limit of small $\delta$ are $\Gamma_{\mathrm{g}}= \pm 0 \cdot 16$. Here, the agreement with the experimental results seems modest; however, one should keep in mind that deviations must be expected since the experiments cannot be performed for very small values of $\delta$. If the exact expression for the transition 


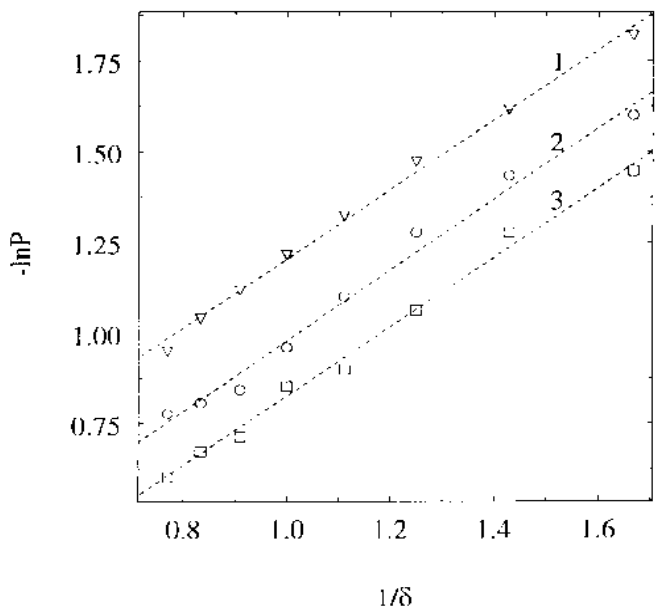

Figure 7. The data points on curve 1 and 3 are the experimental results obtained for the Gaussian twisted Landau-Zener model for opposite signs of the twist function. The parameters are $\alpha=1.00 \mathrm{MHz} \mu \mathrm{s}^{-1}, \Delta=0.223 \mathrm{MHz}, a=1 \mu \mathrm{s}$, and $\mu= \pm 1 \cdot 14 \mathrm{rad}$.

The data points on curve 2 are experimental results for the conventional Landau-Zener model for the same values for $\alpha$ and $\Delta$. The dotted lines are the corresponding numerical simulations.

probability equation (25) is used, the offsets are predicted to be $-0 \cdot 22$ and $0 \cdot 15$, which is in perfect agreement with the experimental results.

The results presented in figure 7 already indicate that the twist function does not give rise to a constant geometric amplitude factor over the full dynamical range. Clearly the transition amplitude will always go to 1 if $\delta$ is large enough. In figure 8 we show the experimental changes in the transition probability due to the twist function over the full dynamical range, i.e., from the adiabatic limit to the diabatic limit. Instead of varying $\delta$ we varied the adiabaticity parameter $\Lambda=\alpha / 2 \pi^{2}$ by changing the coupling strength $\Delta$. In this way, all experimental traces cover an equal time span; this is experimentally much more convenient than changing $\delta$ (we remind the reader that the experiments must be performed within the limited cavity decay time). Besides it is no longer advantageous to plot $-\ln P$ as function of $1 / \delta$ since no straight lines are expected in the diabatic limit. The experimental results are in good agreement with the numerical simulations (dotted curves).

Note that within the present accuracy of the experiment $( \pm 5 \%$ error in the transition probabilities), and for the specific region in parameter space in which the experiments are performed, no differences can be observed between the Gaussian twisted model and the $\phi(\tau)=\beta \tau$ model (recall that the theoretical difference between the transition amplitudes for the two models is less than $3 \%$ ).

We now briefly report an experimental result in the parameter region where three pairs of branch points in the primed frame [shown in figure $4(f)$ ] dominate the final transition probability. In this region, the $\phi(\tau)=\beta \tau^{2}$ model is no longer a proper approximation of the Gaussian twisted LZ model and oscillatory structure is expected in the transition probability as a function of the control parameters. In figure 9 we have plotted the experimental results for a series of measurements of $P$ as a function of $1 / \delta$. Clearly the experimental data points for the Gaussian twisted 


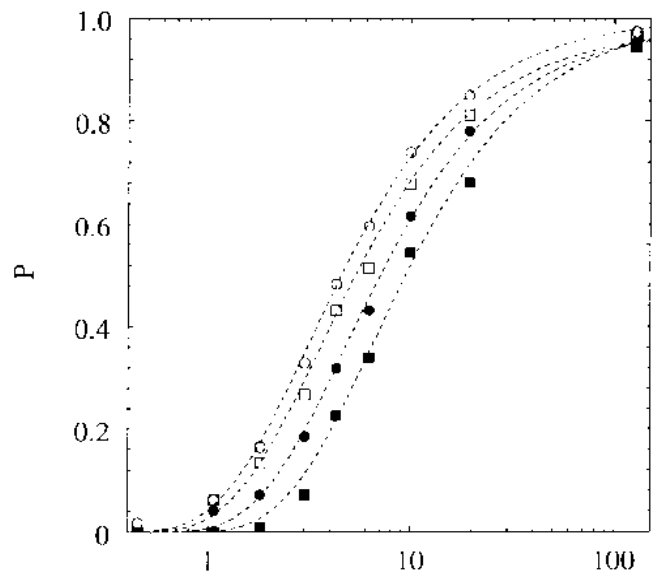

$\Lambda$

Figure 8. Plot of the experimental data points obtained for $P$, as function of $\Lambda=\alpha / 2 \not t^{2}$ for $\alpha=1.28 \mathrm{MHz}_{\mu \mathrm{s}}^{-1}$. The open circular data points are the conventional LandauZener results. The open square, the closed circular, and the closed square data points, respectively, are the results for the Gaussian twisted LZ model with $a=1 \mu \mathrm{s}$, and $\mu=1.5,3.0$, and $4.5 \mathrm{rad}$. The dotted curves show the corresponding numerical simulations.

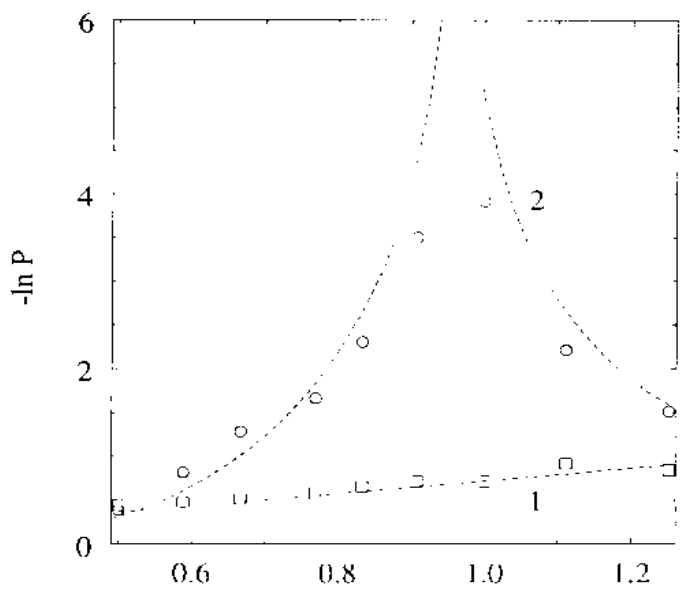

$1 / 8$

Figure 9. The square data points show experimental results for the conventional LandauZener model $\left(\alpha=0.50 \mathrm{MHz} \mu \mathrm{s}^{-1}\right.$, and $\left.\Delta=0.125 \pm 0.01 \mathrm{MHz}\right)$. The circular data points show the experimental results for the Gaussian twisted Landau-Zener model in the parameter region where three pairs of branch points in the primed frame

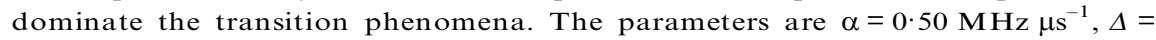
$0 \cdot 125 \pm 0.01 \mathrm{MHz}, a=1 \mu \mathrm{s}$, and $\mu=8.36 \mathrm{rad}$. The dotted curves 1 and 2 show the corresponding numerical simulations. 
LZ model (circles) are not on a straight line parallel to the conventional LZ line (square data point). A distinct maximum in $-\ln P$, i.e. a minimum in $P$, is visible; this indicates the presence of interferences. A quantitative treatment of this situation is outside the scope of the present paper and has been published elsewhere [23].

\section{Conclusions}

We have performed a theoretical and experimental study of the geometric amplitude factor in an optical system. In our presentation, we emphasized the physical insight that is obtained when the Dykhne-Davis-Pechukas method is used in combination with a specific unitary transformation. To perform our experiments we introduced the Gaussian twisted Landau-Zener model which fulfills the physical requirement of finite values for $\phi(\tau)$ as $\tau \rightarrow \pm \infty$. By using this model for small magnitudes of the twist function we verified the theoretical predictions made by Berry [7]. In particular, we observed the geometric amplitude factor in the near adiabatic region and demonstrated that it is independent of the scaled-time parameter $\delta$. For large magnitudes of the twist function we observed the onset of interference phenomena in the transition amplitude due to the interplay of geometric and dynamical effects. The dominant features of such interferences on the transition amplitude is the subsect of a separate paper [23].

For our experiments, we have made use of a classical optical two-level systems for which the quantum-mechanical treatment of two-level systems fully applies. The conventional Landau-Zener model is a very popular model in many branches of physics and chemistry. Therefore, the optically demonstrated phenomena should also be observable in these various branches of science if twist functions are included. For example, in the case of two-level atoms a twist function can be realized by applying additional electro-magnetic fields which are frequencymodulated. Recently, it was shown by Agarwal and Harshawardhan [24] that under this condition population trapping in two-level systems can occur. In the context of the present paper, this population trapping can be seen as a consequence of geometric amplitude effects.

\section{Acknowledgments}

We are grateful to A. Joye for bringing his work to our attention and to M. V. Berry for useful comments concerning the experiments and the manuscript. This work is part of the research programme of the Foundation for Fundamental Research on Matter (FOM) and was made possible by the financial support from the Netherlands Organization for Scientific Research (NWO).

\section{References}

[1] Spreeuw, R. J. C., and Woerdman, J. P., 1993, Progesss in Optics, Vol. 31, edited by E. Wolf (Amsterdam: North-Holland), pp. 263-319.

[2] Spreeuw, R. J. C., Woerdman, J. P., and Lenstra, D., 1988, Phys. Rev. Lett., 61, 318.

[3] Spreeuw, R. J. C., van Druten, N. J., Beijersbergen , M. W., Eliel, E. R., And Woerdman, J. P., 1990, Phys. Rev. Lett., 65, 2642.

[4] Beijersbergen , M. W., Spreeuw, R. J. C., Allen, L., and Woerdman, J. P., 1992 , Phys. Rev., A, 45, 1810.

[5] Bouwmeester, D., Dekker, N. H., van Dorsselaer, F. E., Schrama, C. A., Visser, P. M., and Woerdman, J. P., 1995, Phys. Rev. A, 51, 646.

[6] Schrama, C. A., Boummeester, D., Nienhuis, G., and Woerdman, J. P., 1995, Phys. Rev. A, 51, 641. 
[7] Berry, M. V., 1990, Proc. R. Soc. Lond. A, 430, 405.

[8] Nakamura, K., 1993, Quantum Chaos, Cambridge Nonlinear Science Series 3 (Cambridge University Press).

[9] Nakamura, K., and Rice, S. A., 1994, Phys. Rev. A, 49, R2217.

[10] Joye, A., Kunz, H., and Pfister, Ch.-Ed., 1991, Ann. Phys., 208, 299.

[11] Joye, A., Mileti, G., and Pfister, Ch.-Ed., 1991, Phys. Rev. A, 44, 4280.

[12] Zwanziger, J. W., Rucker, S. P., and Chingas, G. C., 1991, Phys. Rev. A, 43, 3232.

[13] Berry, M. V., 1984, Proc. R. Soc. Lond. A, 392, 45.

[14] Shapere, A., and Wilczek, F., 1989, Geometric Phases in Physics, (Singapore: World Scientific).

[15] Dykhne, A. M., 1962, Sov. Phys. JetP, 14, 941.

[16] Davis, J. P., and Pechukas, P., 1976, J. Chem. Phys., 64, 3129.

[17] Hwang, J.-T., and Pechukas, P., 1977, J. Chem. Phys., 67, 4640.

[18] Landau, L. D., 1932, Phys. Z. Sovjetunion, 2, 46.

[19] Zener, C., 1932, Proc. R. Soc. London. A, 137, 696.

[20] Suominen, K.-A., Garraway, B. M., and Stenholm, S., 1991, Opt. Commun., 82, 260.

[21] Suominen, K.-A., and Garraway, B. M., 1992, Phys. Rev. A, 45, 374.

[22] Clark Jones, R., 1941, J. Opt. Soc. Am., 31, 488.

[23] Bouwmeester, D., Karman, G. P., Schrama, C. A., and Woerdman, J. P., 1996, Phys. Rev. A 53, 985.

[24] Agarwal, G. S., and Harshawardhan, W., 1994, Phys. Rev., A, 50, R4465. 
